\title{
Development of a Portable SLODAR Turbulence Profiler
}

\author{
Richard Wilson ${ }^{\mathrm{a}}$, John Bate ${ }^{\mathrm{a}}$, Juan Carlos Guerra ${ }^{\mathrm{b}}$, Norbert Hubin ${ }^{\mathrm{c}}$, Marc Sarazin ${ }^{\mathrm{c}}$ and Christopher \\ Saunter
}

${ }^{a}$ University of Durham, Dept. of Physics, Rochester Building, South Road, Durham, DH1 3LE, UK ${ }^{b}$ European Southern Observatory, Kart-Schwarzchild-Str. 2, 85748 Garching bei Munchen, Germany ${ }^{c}$ Telescopio Nazionale Galileo, 38700 St Cruz de La Palma, TF-Spain

\begin{abstract}
We report on the development of a prototype portable monitor for profiling of the altitude and velocity of atmospheric optical turbulence. The instrument is based on the SLODAR Shack-Hartmann wave-front sensing technique, applied to a portable telescope and employing an electron-multiplication (EM) CCD camera as the wave-front sensor detector. Constructed for ESO by the astronomical instrumentation group at the University of Durham, the main applications of the monitor will be in support of the ESO multi-conjugate adaptive optics demonstrator (MAD) project, and for site characterization surveys for future extremely large telescopes. The monitor can profile the whole atmosphere or can be optimized for profiling of low altitude $(0-1 \mathrm{~km})$ turbulence, with a maximum altitude resolution of approximately $150 \mathrm{~m}$. First tests of the system have been carried out at the La Palma observatory.
\end{abstract}

Keywords: Turbulence Profile, Seeing, SLODAR, Adaptive Optics, Laser Guide Stars

\section{INTRODUCTION}

Measurements of the vertical profile of optical turbulence, $\mathrm{C}_{\mathrm{n}}{ }^{2}(\mathrm{~h})$, are important in the exploitation of adaptive optics for astronomy in a number of key areas. These include the estimation of anisoplanatism and characterization of the fielddependent corrected point-spread function, optimization of optical conjugation altitudes for deformable mirrors, and optimization of the projection altitude for Rayleigh backscatter laser beacons. Knowledge of the statistical properties of $\mathrm{C}_{\mathrm{n}}{ }^{2}(\mathrm{~h})$ at an observing site is required to predict the imaging performance and observable sky fraction for planned future adaptive optical systems and observations, including the performance of AO corrected imaging with ELTs.

Existing systems for profiling of atmospheric turbulence include balloon micro-thermal probes ${ }^{1}$, and the SCIDAR scintillation profiling method ${ }^{2,3}$. Balloon probes provide high-resolution profiles, but are not suited to continuous monitoring. SCIDAR is a well established technique, but has not been adapted for use as a portable instrument for site characterization.

More recently, the Multi-Aperture Scintillation Sensor (MASS) has been developed as a portable profiler, providing continuous measurements in 6 vertical resolution elements via optical scintillation measurements of a single star ${ }^{4}$. MASS measurements exclude turbulence in the first few hundred meters above the site, which does not contribute to the scintillation.

The SLODAR method is similar to SCIDAR in that it exploits measurements of binary stars to retrieve the turbulence profile via triangulation. The SLODAR profiler employs a Shack-Hartmann wave-front sensor (WFS) to measure local optical phase gradients, whereas SCIDAR measures the scintillation pattern at the telescope aperture. Full details of the SLODAR method are described elsewhere ${ }^{5}$. The normalized turbulence profile is recovered from the cross-correlation of the WFS data for the two stars of the binary target, with a number of resolution elements of the profile equal to the number of WFS sub-apertures mapped onto the aperture of the telescope. The maximum sensing altitude is determined by 
the telescope aperture size and the angular separation of the binary star - closer binaries (or a larger telescope) yield a higher maximum altitude. The integrated turbulence strength is found from the variance of the atmospheric phase aberrations for a single star of the pair. The total contribution from turbulence above the maximum triangulation altitude can also be estimated.

Previously the SLODAR method has been deployed on large astronomical telescopes, including the William Herschel and Mercator telescopes at La Palma ${ }^{6}$. These implementations used wave-front sensors with effective sub-aperture diameters $>10 \mathrm{~cm}$ and exposure times $\sim 5 \mathrm{~ms}$, so that photon rates were high for targets magnitudes $\mathrm{V}<8$. The main technical challenge in adapting the method to a portable telescope $(40 \mathrm{~cm})$ is in achieving good SNR for wave-front sensing with the smaller sub-apertures and exposure times which are demanded when using a smaller telescope. Here the advent of very high sensitivity electron-multiplication (EM) CCD cameras ${ }^{7}$ is key - the effects of read-out noise are overcome, and centroiding of sub-aperture images is possible with only a few tens of detected photons per integration.

An important driver for the development of a portable SLODAR system is that the method can be adapted for profiling of the turbulence in the first few hundred meters above the ground, by using targets with wide angular separations. Low altitude, high-resolution turbulence measurements are required for evaluation of the performance of MCAO and groundlayer adaptive optical corrections using large field-of-view tomography. An important application of the SLODAR monitor will be in support of the ESO multi-conjugate adaptive optics demonstrator system (MAD) ${ }^{8}$. It is envisaged that the system will work in conjunction with MASS and DIMM ${ }^{9}$ monitors in this context.

Here we provide a technical description of the system, and report on the first tests of the prototype, carried out at the Roque de los Muchachos observatory in La Palma in April and May 2004.

\section{TECHNICAL DESCRIPTION}

\subsection{OPTO-MECHANICAL CONSTRUCTION}

The system comprises a Shack-Hartmann wave-front sensor mounted on a Meade $40 \mathrm{~cm}$ Schmidt-Cassegrain telescope (Figures 1 and 2). The WFS is made up of a collimating lens, a lenslet array (manufactured by Adaptive Optics Associates, Inc.), and a short pass filter (550nm cut-off), in a compact tube mounting which is attached directly to the CCD head. Two separate and interchangeable WFS optics sets are used with the system, each of which divides the telescope pupil into an array of $8 \times 8$ sub-apertures. The first is optimized for low altitude profiling using widely separated binary targets $(\sim 60 \mathrm{arcsec})$. In this mode the spot patterns are fully separated on the detector. The second mode is used for more generalized profiling to high altitudes, employing narrow binaries ( $\sim \operatorname{arcsec})$, with the spot patterns interleaved. The camera head and optics are mounted on a powered rotating stage at the Cassegrain focus of the telescope, to permit alignment of the binary position angle relative to the CCD and WFS, and to track the sidereal field rotation (the telescope mounting is alt-azimuth). The target acquisition camera is an Astrovid StellaCam EX integrating video camera with a telephoto lens, mounted on the telescope tube, to provide a 4 degree field of view and with a limiting magnitude of approx. $\mathrm{V}=9$.

The WFS detector is an Andor Ixon DV860B back-illuminated electron-multiplication CCD camera. The detector has a peak quantum efficiency of 92 percent at 550nm, and a maximum EM gain of 1000 times which yields an effective RMS read noise of $<0.1$ electron. Frame rates of up to $450 \mathrm{~Hz}$ (full frame $128 \times 128$ pixels with no binning) are possible. Typically frame rates of approx. $200 \mathrm{~Hz}$ are used for SLODAR, with exposure times of 1-2 ms.

A custom-built portable enclosure has been provided for the system (Fig 3). The octagonal structure is constructed from lightweight panels of anodized aluminium, providing good weather protection. The system can be operated with the enclosure in place and the roof open, or the structure can be completely removed to allow operation in the open air. The enclosure can be disassembled into individual panels for transportation in standard transit cases. The total transit weight of the system, including the telescope and enclosure, is approx $500 \mathrm{Kg}$ in 7 cases. 


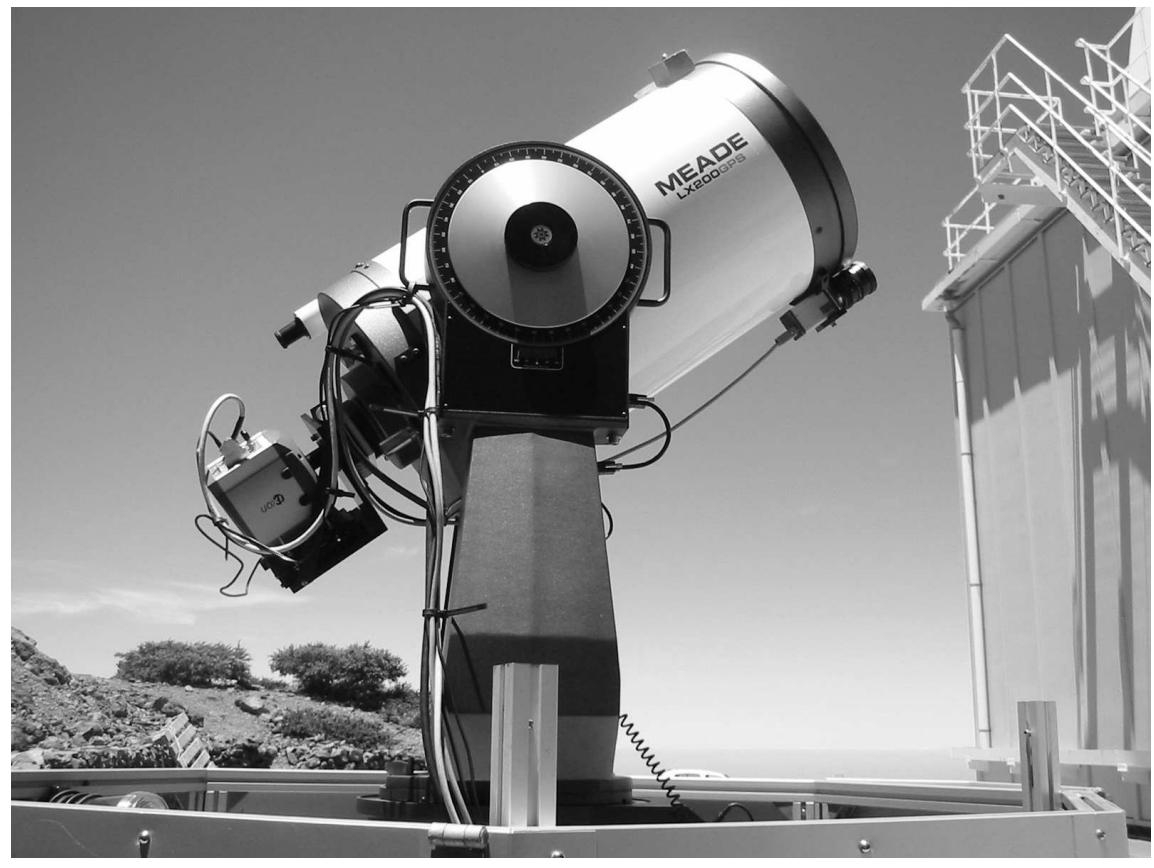

Figure 1. The portable SLODAR turbulence profiler.

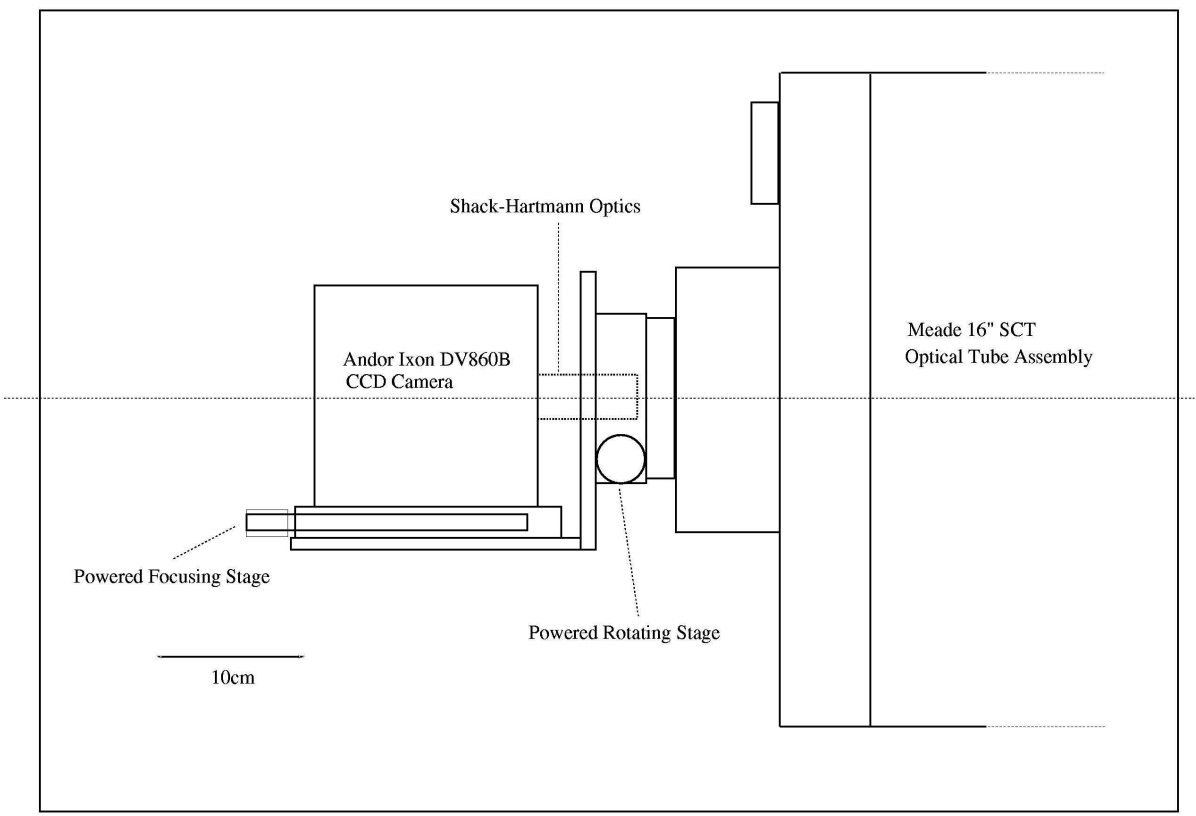

Figure 2. Schematic diagram of the portable SLODAR turbulence profiler system, focal plane instrumentation. 

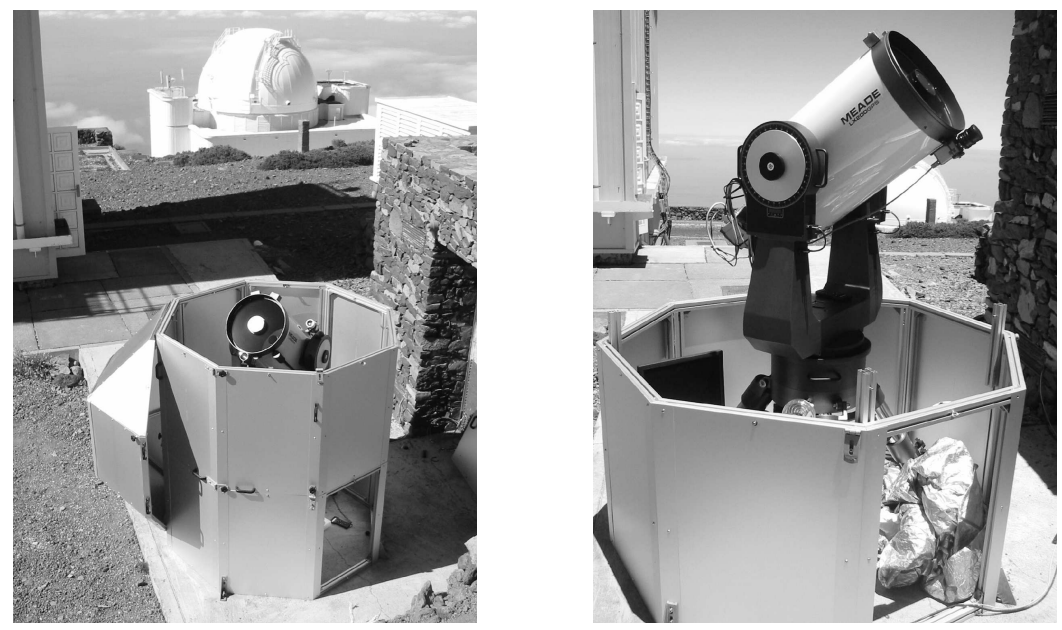

Figure 3. The SLODAR Enclosure.

\subsection{SOFTWARE, CONTROL AND AUTOMATION.}

In the final system, CCD control, data acquisition, real-time reduction, visualization and archiving of the data, as well as telescope, rotator and focus control and target acquisition, will all be performed under Linux on a single dual-processor Pentium 4 PC. Operation of the instrument will be largely automated, with remote control and real-time data display via Ethernet link. Many of the following procedures have already been implemented; full automation is expected by the end of 2004. The control PC selects a target binary star with a suitable RA from a pre-defined list. Target acquisition will be carried out automatically using the integrating video camera to guide the target into the 2 arc-minute field-of-view of the WFS detector. The instrument rotator is set to the known position angle for the chosen binary target, and subsequently tracks the sidereal rotation. To track the target, telescope auto-guiding offsets will be calculated from the WFS camera images.

Once the telescope is tracking, the data acquisition software automatically locates the WFS sub-aperture images on the CCD image and defines a set of centroiding target boxes on the frame. In a typical measurement, 2000 WFS CCD frames are recorded in 10 seconds. Centroids are calculated and passed to the SLODAR data-reduction software for extraction of the atmospheric turbulence parameters (see section 3 ). The value of $r_{0}$, the normalized $\mathrm{C}_{\mathrm{n}}{ }^{2}(\mathrm{~h})$ profile, and a 'movie' of the centroid temporal cross-correlation function (to visualize turbulent layer translation velocities - see figure 8) will be displayed in real time. The raw centroid data and derived turbulence parameters will be archived to disk for later analysis off-line.

\section{RESULTS FROM FIRST TESTING}

\subsection{LIMITING MAGNITUDE FOR WAVEFRONT SENSING}

The portable SLODAR profiler is conceived as an automated device for continuous monitoring of the turbulence parameters, to allow statistical surveys and in support of astronomical observations with AO. Hence there must always be a suitable target binary star available at high elevation $(>50 \mathrm{deg}$ ). In practice this demands a limiting magnitude of approximately $\mathrm{V}=7$ for each component of the binary (although brighter targets are available much of the time). For WFS 
sub-apertures of diameter $5 \mathrm{~cm}$, exposure times of $1 \mathrm{~ms}$ and optical band-pass of 100nm centered at 500nm, a V=7 star will yield $\sim 50$ detected photons per sub-aperture per integration. Typically a $6 \times 6$ or $8 \times 8$ pixel sub-region of the CCD is required for centroiding of each sub-aperture. Hence for a conventional CCD with at least 3electrons rms read noise per pixel, the SNR for centroid measurements would be very low at such light levels. The SLODAR system, equipped with the electron-multiplication CCD, has been operated effectively using a binary target with component magnitudes $V=6.2$ and $V=7.2$. Hence the limiting magnitude of the system is sufficient to achieve continuous monitoring. Note that measurement of the turbulence profile from the centroid cross-correlation is not biased by the effects of photon noise, since different photons are involved in the wave-front sensing for each star.

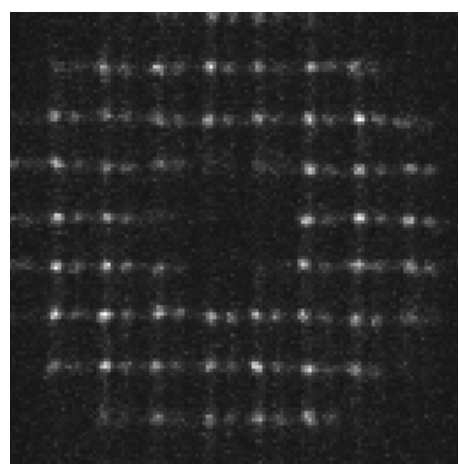

Figure 4. Example WFS EM-CCD frame for a binary target with component magnitudes V=5.1 and V=6.0 ( $1 \mathrm{~ms}$ exposure).

\subsection{FRIED'S PARAMETER}

The integrated turbulence strength, in terms of Fried's coherence length parameter $\mathrm{r}_{0}$, is determined from the variance of the Zernike coefficients of the measured phase aberration, by fitting to the expected distribution for Kolmogorov turbulence ${ }^{10}$. The mean image motion, averaged over all sub-apertures, is excluded from the analysis so that the measurement is not contaminated by telescope wind-shake and guiding errors. A value of $r_{0}$ can be calculated for each of the two target stars, as a check of consistency and the SNR for wave-front sensing on each component. Figure 5 shows an example of the Zernike variance distribution and $r_{0}$ determination from the testing at La Palma.
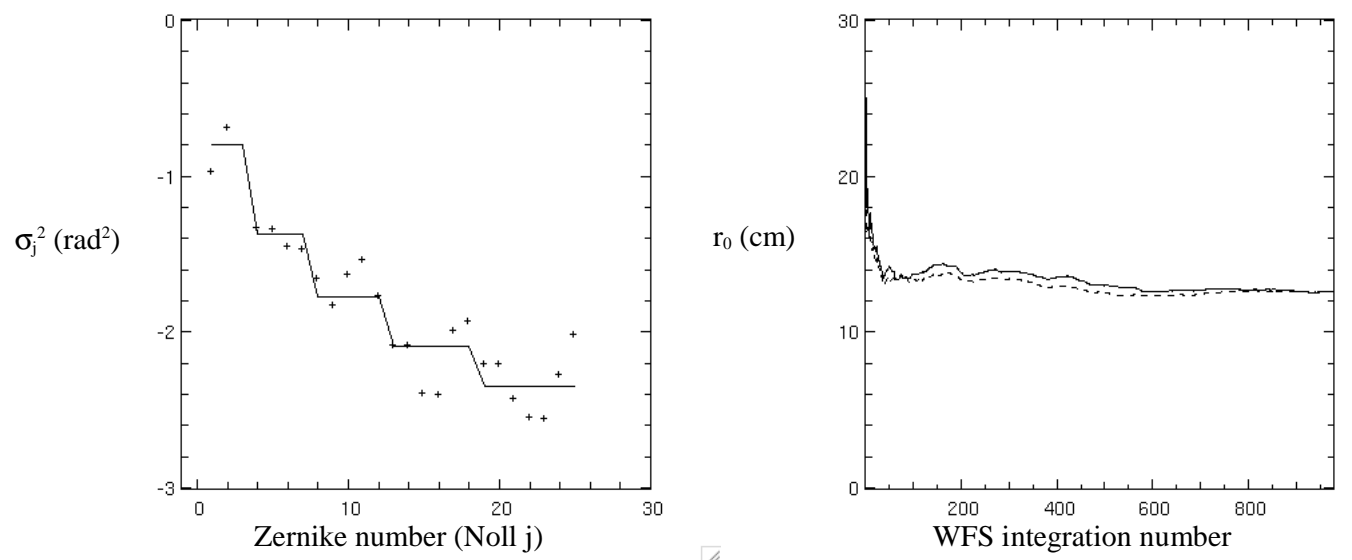

Figure 5. An example SLODAR determination of $r_{0}$ for April 6, 2004. Left: Measured Zernike coefficient variances, $\sigma_{j}^{2}$ (crosses) and the theoretical (Noll) fit (solid line). Right: Calculated value of $\mathrm{r}_{0}$ versus WFS integration number (2ms CCD integrations at $190 \mathrm{~Hz}$ ). 


\subsection{TURBULENCE PROFILES}

The normalized turbulence profile is recovered from the spatial cross-correlation of the WFS (centroid) data for the two stars. The rotator is used to align the rows of the WFS sub-apertures along the vector separation of the target binary. Then the correlation need only be calculated in one spatial dimension to yield the profile. The auto-correlation of the centroids for one star of the pair is used as a measure of the 'impulse response' of the system to a single turbulent layer, so that the normalized vertical turbulence profile can be deconvolved from the cross-correlation function.

Figure 6 shows example measured correlations and turbulence profiles from testing of the SLODAR system at La Palma on the night of May 17-18, 2004. On the left are plots for data recorded at UT 23:22, and on the right for data recorded later on the same night, at UT 01:43 on May 18. The data were recorded for a binary target with separation 6 arcsec, so that the maximum profiling altitude was $12 \mathrm{~km}$, with a vertical resolution of $1.7 \mathrm{~km}$.

In both examples there is strong turbulence at close to ground level, indicated by the peak in the cross-correlation at zero spatial offset. At 23:22 there is also significant turbulence at higher altitudes, evidenced by the asymmetry of the crosscorrelation function, with excess correlation values at positive offsets (corresponding to positive altitudes) relative to negative offsets (corresponding to 'virtual' or negative altitudes which should contribute no turbulence). Two distinct contributions are resolved in the deconvolved profile, at altitudes of approximately $4 \mathrm{~km}$ and $9 \mathrm{~km}$. By 01:43 these higher altitude contributions have subsided, so that the shape of the cross-correlation is almost identical to that of the autocorrelation (middle-right panel). Hence the profile is dominated by low-level turbulence. Note however, that the peak height of the cross-correlation is lower than that of the auto-correlation (top-right panel), indicating that there is a significant turbulence $\left(\sim 30\right.$ percent of the total integrated $\left.C_{n}{ }^{2}(h)\right)$ at altitudes above the maximum profiling altitude $(12 \mathrm{~km})$. This then contributes to the auto-correlation but not to the cross-correlation.

\subsection{TURBULENCE VELOCITY}

The temporal characteristics of the optical turbulence can also be measured from the WFS data. For example, power spectra of the measured Zernike coefficients can be calculated, or a value for the coherence time, $\mathrm{t}_{0}$, can be derived. As well as altitudes, translational velocities for individual turbulent layers can be determined from the spatio-temporal crosscorrelation of the centroid data for a binary target. This is demonstrated in figure 7 which shows the two dimensional cross-correlation for sample data from commissioning tests at the ORM on the night of May 16, 2004. The data are plotted for increasing time offset $\delta \square$ between the data streams for the two stars of the target. In the left-most panel, $\delta \mathrm{t}=0$, a strong signal can be seen in the centre of the autocorrelation, corresponding to turbulence at close to ground level (for a higher altitude layer the corresponding peak would be displaced to the right of center on the plot for $\delta \mathrm{t}=0$ ). In the subsequent frames, with increasing $\delta \mathrm{t}$, the peak moves away from the center, indicating a translational velocity of $10 \mathrm{~m} / \mathrm{s}$ for the turbulent layer. This corresponds closely to the ground-level wind speed measured by the observatory meteorological station at the time of the observation.

Non-translational temporal development of a turbulent layer can also be characterized from the spatio-temporal crosscorrelation: wind-shear effects will be seen as an elongation or distortion of the correlation peak with increasing $\delta \mathrm{t}$; 'boiling' or non-Taylor development of the layer would be manifest as a gradual decrease in the total correlation associated with the layer (the summed 'flux' within the spot on the correlation plot) with increasing time offset. 

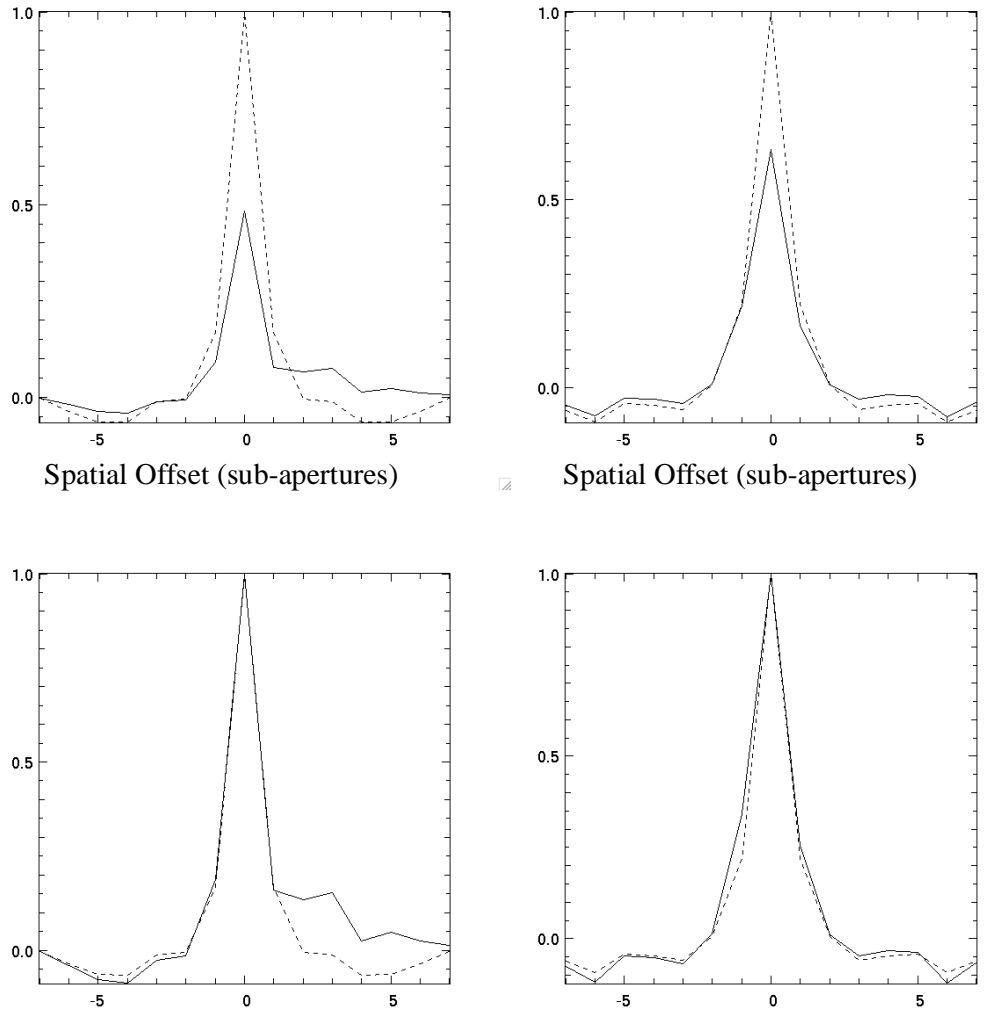

Spatial Offset (sub-apertures)

Spatial Offset (sub-apertures)
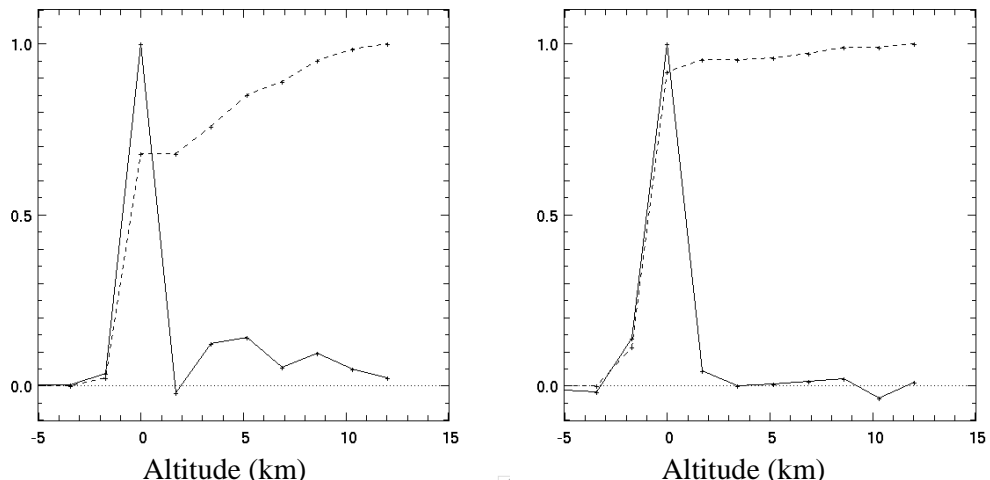

Figure 6. Example normalized cross-correlation functions and deconvolved turbulence profiles measured on May 17, 2004 (see text). Left: UT 23:22; Right: UT 01:43 (May 18). Top: Cross-correlation (solid line) and autocorrelation (broken line) functions normalized to the peak value of the auto-correlation. Middle: The same cross-correlation and auto-correlation functions, each normalized to a peak value of 1 to allow comparison of the shape of the functions. Bottom: Deconvolved $\mathrm{C}_{\mathrm{n}}{ }^{2}(\mathrm{~h})$ profiles, normalized to a peak value of 1 (solid line ) and the cumulative profile (broken line). The integrated $r_{0}$ values measured in each case were $11.0 \mathrm{~cm}$ at $23: 22$ and $11.9 \mathrm{~cm}$ at $01: 43$. 

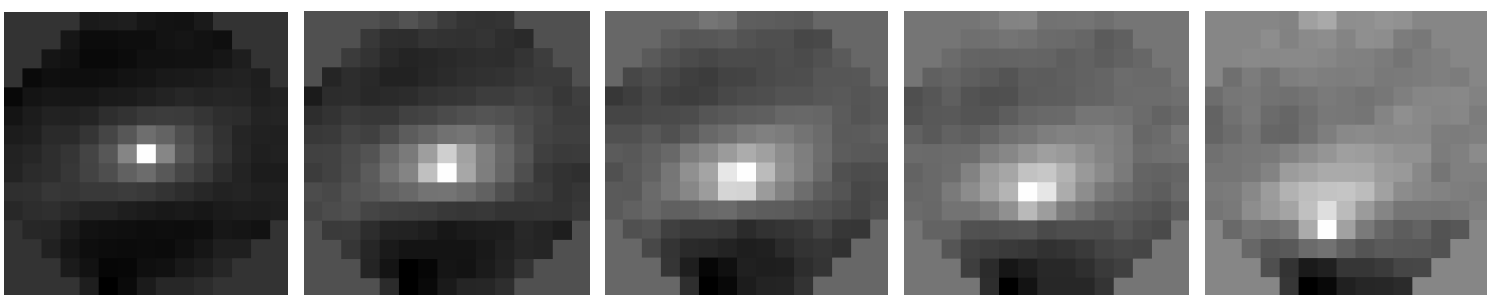

Figure 7. Example two dimensional centroid spatio-temporal cross-correlation function, with increasing temporal offset $\delta$ t between the data streams for the two stars of the target binary (see text). Left to right: $\delta \mathrm{t}=0$, increasing in $5 \mathrm{~ms}$ steps to $20 \mathrm{~ms}$. The movement of the correlation peak is associated with a translational velocity of the low-altitude turbulence of $10 \mathrm{~m} / \mathrm{s}$.

\section{SUMMARY}

A prototype portable system for profiling of the altitude and velocity of optical atmospheric turbulence is being developed, based on the SLODAR technique. Successful first field tests of the instrument have been carried out at La Palma. Full automation of the system as a routine seeing and turbulence monitor is planned for the end of 2004.

\section{ACKNOWLEDGEMENTS}

The authors are grateful to the staff of the Isaac Newton group and the Instituto de Astrophysica de Canarias for their assistance in facilitating field tests at the ORM, and for financial support from ESO and the UK PPARC (RWW). We thank Tim Butterley, Tim Morris and Paul Clark for technical assistance.

\section{REFERENCES}

1. Vernin J., Munoz-Tunon, C., "Optical seeing at the La Palma Observatory”, A\&A 284, 311, 1994.

2. Kluckers V.A., Wooder N.J., Nicholls M.J., Munro I., Dainty J.C., "Profiling of atmospheric turbulence strength and velocity using a generalized SCIDAR technique", A\&AS 130, 141, 1998.

3. Avila R., Vernin J., Sanchez L.J., "Atmospheric turbulence and wind profile monitoring with generalized SCIDAR”, A\&A 369, 364, 2001.

4. Kornilov, V.K., et al., "MASS: a monitor of the vertical turbulence distribution", proc. SPIE 4839, 837, 2003.

5. Wilson R.W., "SLODAR: Measuring optical turbulence altitude with a Shack--Hartmann wave-front sensor", MNRAS 337, 103, 2002.

6. Wilson, R.W \& Saunter, C.D., "Turbulence profiler and seeing monitor for laser guide star adaptive optics", proc. SPIE 4839, 446, 2003.

7. Basden, A.G., Haniff, C.D. \& Mackay, C.D., "Photon counting strategies with low light level CCDs", MNRAS 345, 985, 2003.

8. Marchetti, E., et al., "MAD: the ESO multi-conjugate adaptive optics demonstrator", proc SPIE 4839, 317, 2003.

9. Sarazin, M. \& Roddier, F., “The ESO differential image motion monitor”, A\&A 227, 294.

10. Noll, R., “Zernike polynomials and atmospheric turbulence”, JOSA 66, 207, 1976. 